\title{
Classifying leukemia types with chromatin conformation data
}

\author{
Mathieu Rousseau ${ }^{1,2+}$, Maria A Ferraiuolo ${ }^{1 \dagger}$, Jennifer L Crutchley $^{1+}$, Xue Qing David Wang ${ }^{1}$, Hisashi Miura ${ }^{1}$, \\ Mathieu Blanchette $2^{2^{*}}$ and Josée Dostie
}

\begin{abstract}
Background: Although genetic or epigenetic alterations have been shown to affect the three-dimensional organization of genomes, the utility of chromatin conformation in the classification of human disease has never been addressed.

Results: Here, we explore whether chromatin conformation can be used to classify human leukemia. We map the conformation of the HOXA gene cluster in a panel of cell lines with 5C chromosome conformation capture technology, and use the data to train and test a support vector machine classifier named 3D-SP. We show that 3D-SP is able to accurately distinguish leukemias expressing MLL-fusion proteins from those expressing only wild-type MLL, and that it can also classify leukemia subtypes according to MLL fusion partner, based solely on 5C data.
\end{abstract}

Conclusions: Our study provides the first proof-of-principle demonstration that chromatin conformation contains the information value necessary for classification of leukemia subtypes.

\section{Background}

The organization of the human genome in the nucleus is non-random and important for proper gene expression [1-4]. For instance, chromosomes are known to occupy distinct regions called 'chromosome territories' that are anchored to the lamina. Gene-rich chromosomes tend to localize at the center of the nucleus and gene-poor near the periphery [5-9]. The co-localization of co-regulated genes into transcription factories is another key feature of genome organization that is thought to coordinate and/ or potentiate transcriptional responses [5-9]. Chromatin architecture can also control transcription by promoting or restricting physical proximity between distal control DNA elements. As such, long-range contacts can correlate with either activation or repression of transcription [10-12]. For example, transcription activation at the $\beta$-globin locus associates with physical contacts between the locus control region (LCR) and actively transcribed genes [13]. Conversely, insulator (CTCF)-mediated loops differentially compartmentalize inactive genes away from enhancers at

\footnotetext{
* Correspondence: blanchem@cs.mcgill.ca; josee.dostie@mcgill.ca ${ }^{\dagger}$ Equal contributors

${ }^{2}$ McGill Centre for Bioinformatics and School of Computer Science, McGill University, Montréal, Québec H3G OB1, Canada

'Department of Biochemistry and Goodman Cancer Research Center, McGill University, Montréal, Québec H3G 1Y6, Canada
}

the apolipoprotein locus [14]. The relationship between architecture and expression is also well illustrated by the inactivation of one $\mathrm{X}$ chromosome in the nuclei of female mammalian cells [15], where epigenetic silencing leads to a condensed chromatin structure with silent genes at the core and expressed ones looped out [16].

Given the relationship between genome architecture and activity, it is not surprising that human disease can sometimes be attributed to defects in genome organization. Compelling evidence for the role of three-dimensional (3D) chromatin organization in human disease comes from studies on laminopathies like the Emery-Dreifuss muscular dystrophy. It was shown that this disease could originate from mutations in a lamin protein that specifically causes abnormal retention and silencing of muscle-specific genes at the nuclear envelope [17]. The importance of spatial genome organization in human disease is equally well demonstrated in cancers where single nucleotide polymorphisms (SNPs) were found to create novel enhancers acting long-range to activate distal genes through DNA looping [18-20].

Overall, genome architecture is guided by chromatin interactions with nuclear landmarks like the lamina, and by intra- and inter-chromosomal contacts mediated by chromatin-binding proteins. In addition to transcription 
factors that form DNA loops between enhancers and promoters, proteins like CTCF, SATB1, and the Cohesin complex are thought to be master regulators of genome organization. Protein complexes such as those containing Polycomb group proteins or the mixed lineage leukemia (MLL) protein might be equally important in shaping the human genome [21-23]. MLL is an H3K4 methyltransferase that is present in COMPASS-like (complex of proteins associated with Set1) complexes [24]. These multi-subunit complexes are very large, and activate transcription partly by methylating H3 on Lys 4 . COMPASS-like complexes control the expression of many genes with pivotal roles in development and differentiation including homeobox family members like the HOX genes.

The MLL gene is a common target in non-random chromosomal translocations associated with both acute lymphoblastic (ALL) and acute myeloid (AML) leukemia, with over 50 different translocation partners identified so far [25-27]. These translocations result in the production of gain-of-function chimeras composed of an aminoterminus MLL lacking the SET domain fused in frame with another protein coding gene. In all cases, the resulting MLL fusion oncoprotein acts as a strong transcriptional activator that disrupts the normal hematopoietic differentiation program by inducing the aberrant expression of key regulators including $H O X$ family members [28]. In fact, dysregulation of $H O X$ genes was reported to be a dominant mechanism of leukemic transformation by MLL fusions [29].

The mechanisms by which different MLL fusions activate transcription or lead to either AML or ALL are poorly understood. However, the observation that many translocation partners are elongation factors that co-exist in a super elongation complex (SEC) with the fusion proteins suggests that they can activate transcription at the elongation step [30]. Interestingly, many fusion partners of MLL bind each other to form transcription foci visible by microscopy. Since the expression of MLL chimeras can alter their localization and activity, these findings suggest that MLL fusions might alter chromatin organization $[22,31,32]$. Also, given that the epigenetic state of chromatin and its conformation are thought to mutually affect each other in self-enforcing structure-function feedback loops [33], it seems likely that MLL fusions additionally alter chromatin organization by modifying its epigenomic profile.

In infants, MLL is translocated in over $50 \%$ of acute leukemias [34,35], whereas translocations in adults are most often seen in patients having undergone chemotherapy [36]. In general, the prognosis of AML patients expressing MLL fusions is poor [37], and new classification methods could help identify optimal treatment courses. We previously reported that terminal differentiation of the AML
THP-1 cell line into macrophages is accompanied by transcriptional repression and spatial remodeling of the HOXA gene cluster [38]. From these results, we wondered whether chromatin architecture, which in essence reflects genome activity, could be used to classify leukemia types. Here we present a study that provides a proof of concept that chromatin conformation can be used to classify leukemias. We found that when the highly relevant HOXA region is considered, chromatin architecture has the information value to distinguish between leukemia types and subtypes.

\section{Results and discussion}

\section{The HOXA gene cluster as a test locus}

The HOX clusters encode transcription factors that are important for embryonic development and hematopoietic lineage regulation $[39,40]$. Aberrant $H O X$ expression is found in various types of human cancers including lung cancer [41], breast cancer [42], melanoma [43], and leukemia [44]. HOXA9 and 10 for instance are oncogenes overexpressed in various leukemia types and are direct targets of MLL fusion oncoproteins [45-47]. In mammals, there are $39 H O X$ genes organized into 13 paralogue groups and divided into four clusters named $A, B, C$, and $D$ located on different chromosomes $[48,49]$. The human HOXA cluster spans over $100 \mathrm{kbp}$ on chromosome 7 and encodes 11 transcription factors (Figure 1A). To determine whether chromatin architecture can be used to classify disease, we mapped the organization of a region containing the HOXA cluster with the chromosome conformation capture carbon copy (5C) technology (Figure 1B). The $5 \mathrm{C}$ method is a member of the so-called ' $3 \mathrm{C}$ technologies' used to measure genome organization in vivo at highresolution [50,51]. 5C captures chromatin conformation by converting chemically cross-linked chromatin segments into unique ligation products, which are then detected high-throughput using a modified version of ligation mediated amplification (LMA).

Using an experimental design previously described [38] we measured chromatin contacts throughout the HOXA cluster region in a panel of leukemia cell lines (Figure 1C). This panel, which is detailed in Additional file 1: Supplementary Materials and methods, includes 20 samples expressing MLL fusions and 10 with only the $w t$ protein (Figure 1D, left). The panel featured AML and ALL caused by a fusion between MLL and the AF9 gene (MLL-AF9; AML), MLL and the ENL gene (MLL-ENL; ALL), or expressing the wild-type $(w t)$ protein (Figure 1D, right). We also included three embryonic carcinomas (EC) samples in this training set to increase diversity. These are known to encode only the wt MLL protein and express no HOXA genes ([52] and Additional file 2: Table S1). The normalized 5C data from these samples were derived as detailed in Additional file 1: Supplementary Materials and methods and shown 


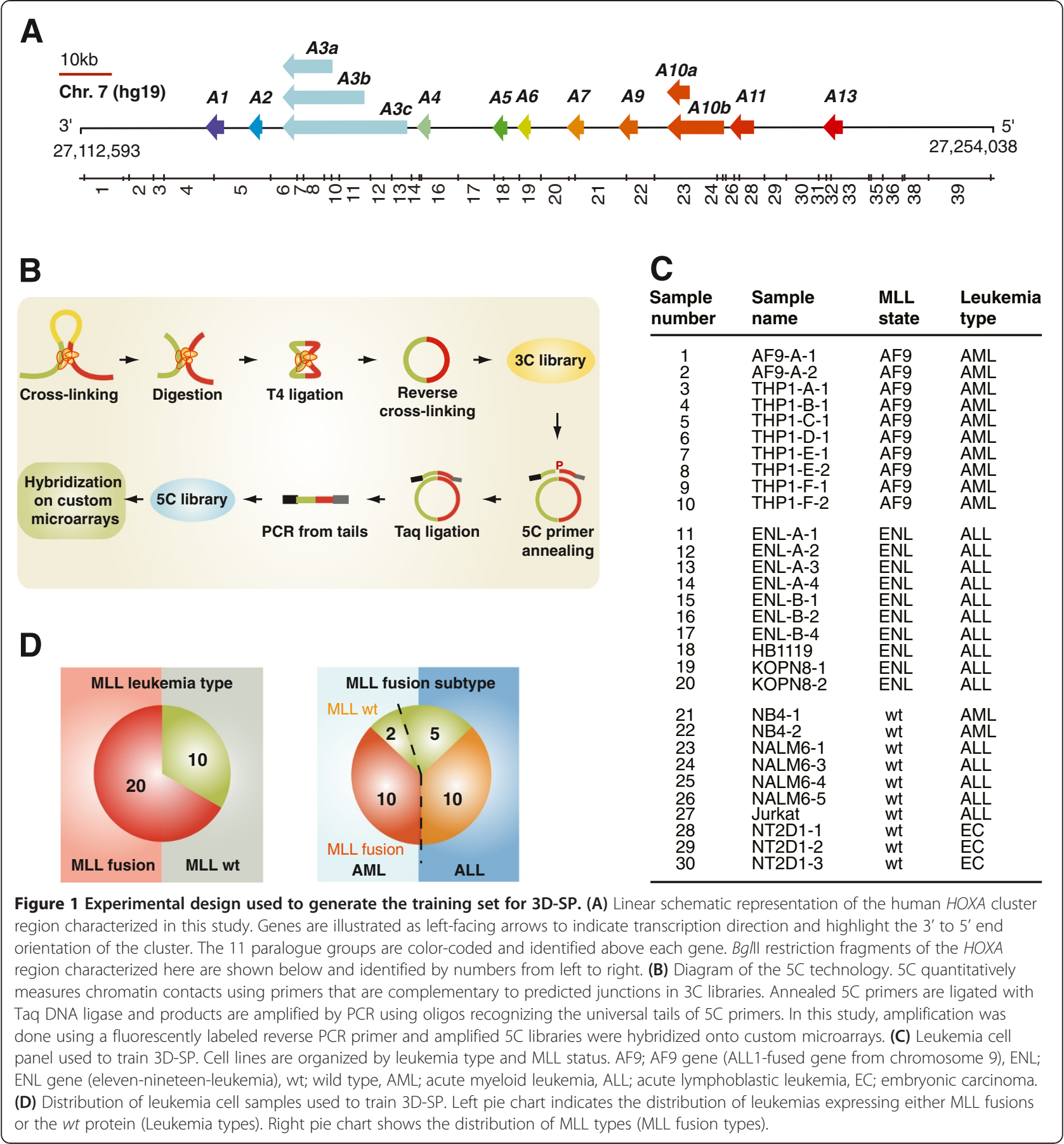

in Additional file 3: Figure S1. The 5C datasets are presented in heatmap form in Additional file 4: Figures S2, and show a very high degree of variability between all samples, regardless of whether they express MLL fusions, the $w t$ protein are AML or ALL. When comparing the average HOXA interaction frequencies (IFs) in leukemia samples expressing MLL fusions to those encoding only $w t$ MLL, we could find marked differences in contact frequencies between neighbors (heatmap diagonal) and between distal fragments interacting long-range (Figure 2A). For example, we observed higher local contacts around the HOXA3 gene in MLL fusion samples, and more longrange interactions between the 5 ' end, the middle and the 3 ' end of the cluster in samples where only the wt MLL protein is expressed (Figure 2A, right). These results indicate that the HOXA chromatin conformation in leukemia cell lines expressing MLL fusions and MLL wt might differ sufficiently to be used for classification. 


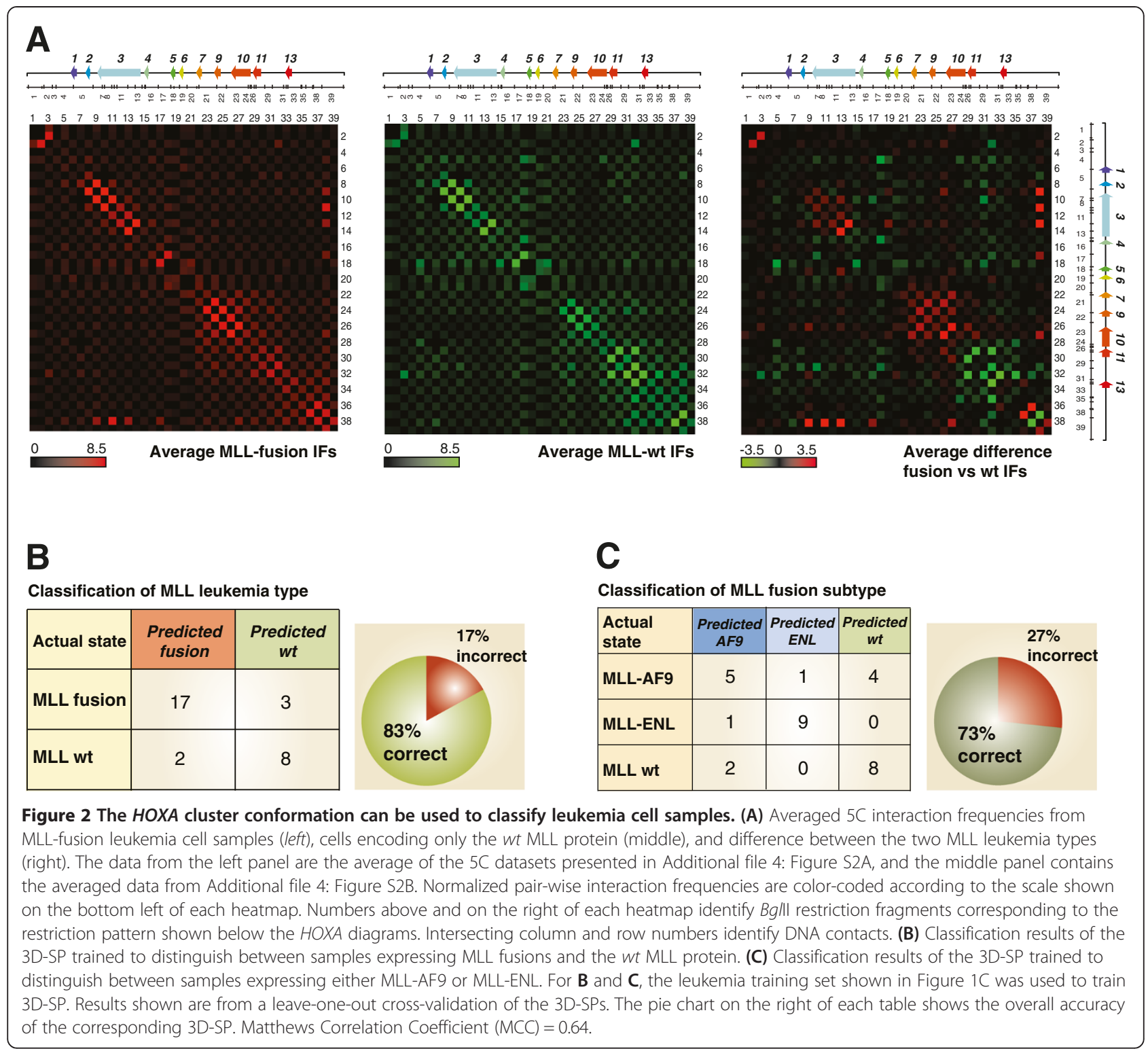

\section{Development, training, and performance of 3D-SP}

Although significant differences could be observed between averaged IFs, this type of 'direct' measurement does not reliably identify contacts that most consistently describe a particular leukemia type and that could be used for classification. Indeed, average or greater IFs in a given sample set might simply originate from the presence of a few samples where these contacts are high. To more robustly distinguish between leukemia types, we developed a support vector machine (SVM; [53]) classifier called '3DSP' (3-Dimensional DNA Disease-Signature Predictor), which uses the complete IF data from a $5 \mathrm{C}$ experiment as basis for classification. We opted for an SVM since they were previously shown to yield good accuracy classifiers even for high-dimensional data [54].
3D-SP was evaluated using leave-one-out cross-validation on the set $S$ of 30 samples shown in Figure 1C. Specifically, for each sample $s$ in $S$, a classifier was trained on the 29 remaining samples $(S-\{S\})$ and then used to predict the class of $s$. The result of this cross-validation procedure is then reported as one entry in the confusion matrix shown in Figure 2B. This ensures that no predictor was trained using the sample on which it is asked to make a prediction. Using this approach, we found that leukemia samples expressing either MLL fusions or the $w t$ protein could be classified with $83 \%$ accuracy by 3D-SP (Figure 2B). Training 3D-SP to recognize features specific to MLL fusion subtypes also yielded good classification results by leave-one-out cross-validation albeit with a lower accuracy of $73 \%$ (Figure 2C). These results demonstrate 
that the HOXA cluster organization can be used to classify different leukemia types.

\section{Identifying highly predictive chromatin contacts}

We next wondered which HOXA contacts showed the greatest difference between classes and conferred the largest amount of predictive power in the classification of leukemias expressing either MLL fusions or the $w t$ protein. By measuring the information gain score of each pair-wise interaction, we found that over 20 different contacts contributed information that enhanced the classification performance (Figure 3A; Student t-test $P$ value $<0.01$ ). The information gain estimates the reduction of entropy in the classification achieved by each contact, and can therefore be used to identify discriminatory interactions. As expected, there were much fewer contacts than those displaying large averaged IFs differences (compare Figures 2A and $3 \mathrm{~A})$. For instance, the predictor did not retain most neighboring interactions, which were strong IF values that differed greatly between leukemia sets.

Interestingly, we observed a significant difference between the average IF values of informative contacts in leukemias expressing MLL fusions compared to the $w t$ protein (Figure 3B). Specifically, we found that a region downstream of HOXA13 at the cluster 5' end preferentially interacts with its 3' part in wt MLL samples (Figure 3C, fragments 31 to 35). In contrast, more contacts were observed between the HOXA11 gene (fragments 26 and 27) and the cluster, suggesting that these two regions are differentially regulated in leukemias expressing MLL fusions. This result was interesting in light of our previous report that differentiation of THP-1 promyelomonocytic leukemia cells into macrophages is accompanied by transcription repression of 5' end genes and the formation of long-range contacts between the ends of the cluster [38]. Given that MLL fusions appear to alter organization, perhaps by modifying the chromatin at specific regions along the cluster, this result might also provide insight on how the fusions activate transcription. Whether DNA sequences at the HOXA11 and HOXA13 regions are important for the observed conformational changes is unclear but mapping of CTCF and cohesin by ChIPseq shows that the two proteins bind to these regions (Figure 3C, bottom). CTCF and cohesin are known to form long-range interactions and it will be interesting to see whether their association with the chromatin or binding to each other to form loops are specifically targeted by MLL fusions.

In a similar manner, we looked for HOXA contacts showing the greatest informative differences between the MLL fusion subtype classes (Figure 4). For this, we measured the information gain value of each feature for the subtype prediction task and found 20 contacts with significant predictive value (Student t-test $P$ value $<0.01$ )
(Figure 4A). In contrast to the predictive features of MLL leukemia types, the contacts distinguishing MLL-ENL from MLL-AF9 leukemias were distributed throughout the cluster (Figure 4B,C) and did not particularly cluster at sites bound by CTCF and cohesin. These were generally stronger in MLL-AF9 samples, except for 14-15 and 32-33 that were also identified as good predictors of $w t$ MLL samples. We do not think that stronger contacts in MLLAF9 can be explained simply by more expression of the cluster in either of the sample sets since each featured high and low expressers (Additional file 2: Table S1). Also, transcriptional activity does not appear to be a defining parameter in classification (see below and Additional file 5: Figure S4). Thus, we favor a model whereby different MLL fusions lead to distinct chromatin conformations by specifically recruiting proteins and modifying the chromatin at the cluster.

De novo classification of MLL leukemia samples with 3D-SP All the analyses with 3D-SP presented above were performed using a leave-one-out cross-validation approach and we wanted to confirm that the classifier would generalize to new samples. To this end, we generated $5 \mathrm{C}$ interaction maps for a test leukemia cell panel (Figure 5A and Additional file 6: Figure S3), and used the 3D-SP previously trained to distinguish between MLL $w t$ and fusions with the training set (Figure $1 C$ ) to classify these data. The test leukemia set included leukemia cell lines expressing MLL-AF6, MLL-AFX, and MLL-AF4 and a new cell line expressing the MLL-AF9 fusion. Cell lines encoding $w t$ MLL included AML, ALL, and the EC cell line NT2D1 induced with retinoic acid for $24 \mathrm{~h}$. We added this sample because although it does not express an MLL fusion, the 3' end genes are expressed and we expect the cluster to adopt an open configuration [52]. We found that 3D-SP classified the test leukemia cell lines expressing MLL fusions or wt MLL with perfect accuracy. Furthermore, 3D-SP also correctly classified five biological replicates of the MLL-AF9 expressing THP-1 samples produced in another study [55]. Even the EC sample expressing 3' end HOXA genes was correctly classified suggesting transcription and opening of the cluster were not determining parameters in the classification by $3 \mathrm{D}-\mathrm{SP}$.

Indeed, transcription activity at the cluster did not seem to be a deciding factor in the classification since three of the four cell lines expressing MLL wt (HL60, U937, MOLT-4) expressed significant levels of HOXA genes, while one of the MLL fusion leukemia cell lines (Karpas-45) did not express the genes at all and yet, all were correctly classified (Additional file 2: Table S1). In fact, we found that $5 \mathrm{C}$ performed much better than gene expression when we compared SVM classification of a representative cell panel based on HOXA9 expression 


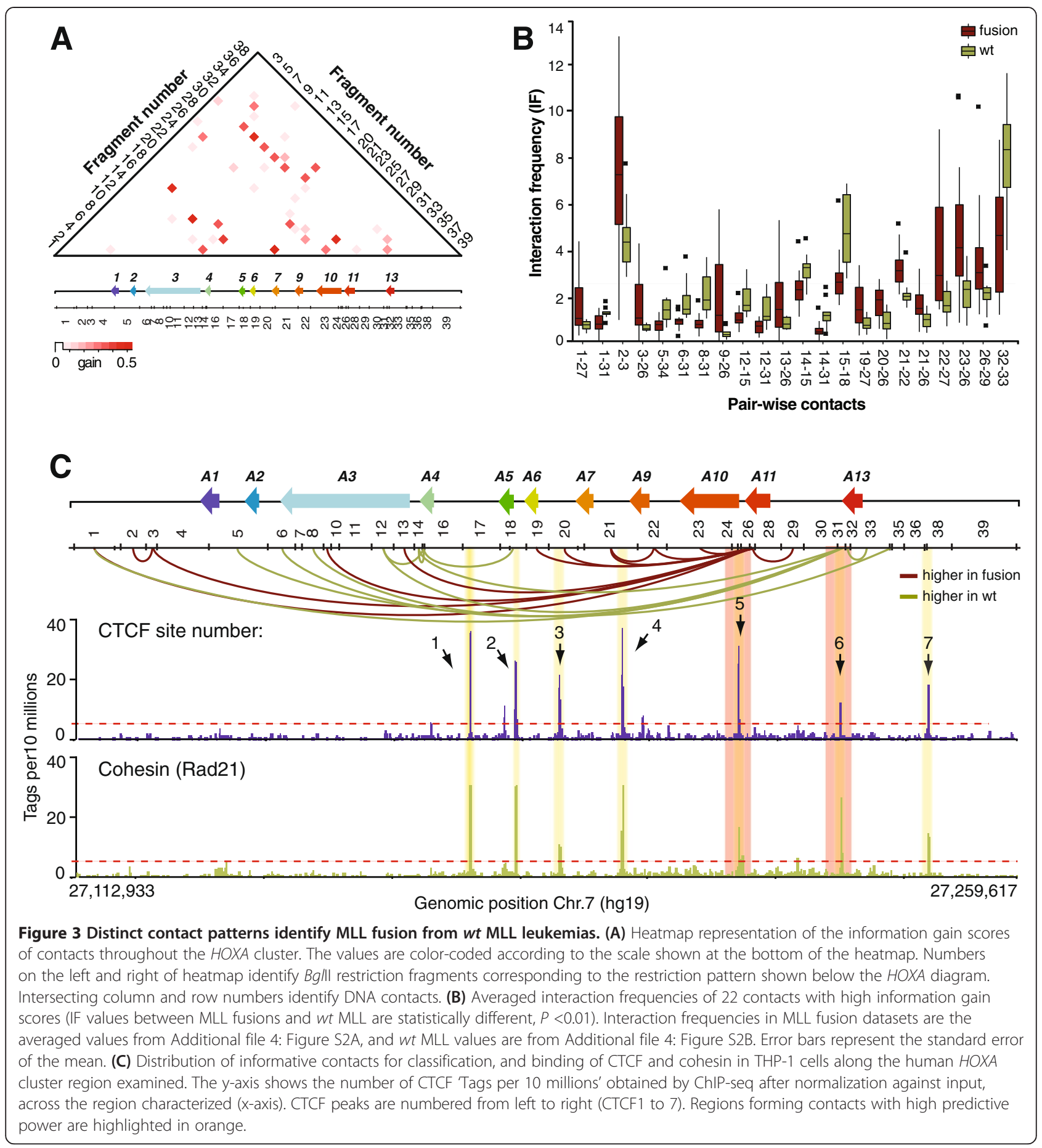

(48\%), all HOXA gene expression $(62 \%)$ or on the $5 \mathrm{C}$ data (93\%; Additional file 5: Figure S4B and C). Prediction based on gene expression was improved when a decision tree classifier was used instead of an SVM (HOXA9; 86\%, all HOXA; 83\%) but remained slightly lower than $5 \mathrm{C}$ classification with an SVM (Additional file 5: Figure S4D). Although our data do not definitively show that chromatin conformation is more robust than gene expression, $5 \mathrm{C}$ data do appear to contain additional information not present in gene expression datasets that improve classification. Together, these results provide the very first proof of principle that $3 \mathrm{D}$ chromatin organization of the HOXA cluster can be used to classify MLL fusion leukemia cell types. 


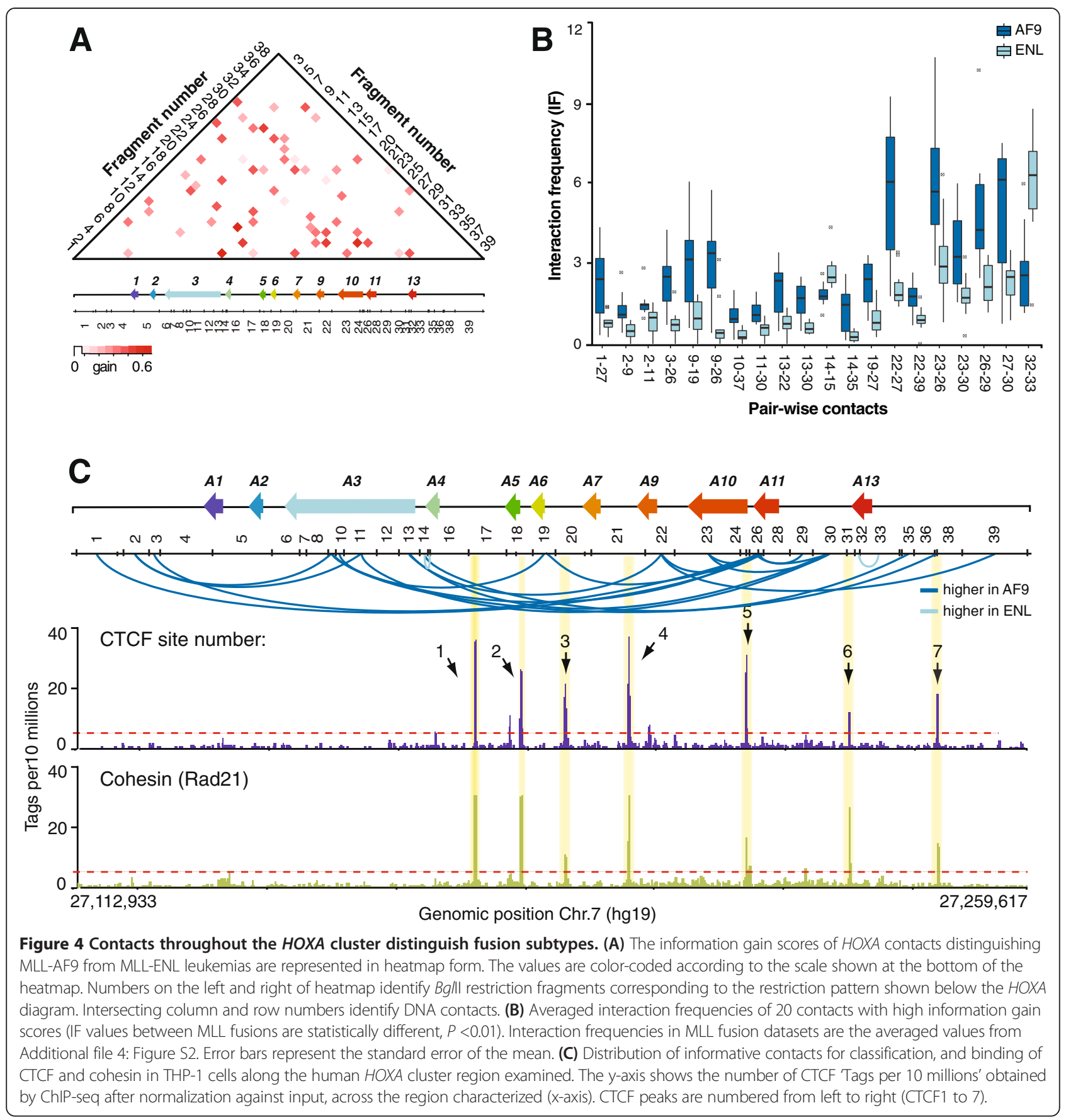

\section{Conclusions}

3D chromatin organization represents a type of higherorder transcription regulation mechanism used to control gene expression in a tissue-specific manner. Here, we provide evidence that chromatin conformation can be used to classify leukemia samples. We found that contacts from at least two regions along the HOXA cluster significantly contribute to the classification. The first region localizes at the 3 ' end of the HOXA11 gene and forms more contacts with the cluster when MLL fusions are expressed, while the second region lies downstream of the HOXA13 gene and forms more contacts with the cluster in samples encoding the $w t$ protein. These results suggest that these two regions are differently regulated in leukemias expressing MLL fusions, and one would expect that proteins interacting with one or more of these sequences would be responsible for these interactions. As described in the results section and discussed further 
A

\begin{tabular}{|c|c|c|c|}
\hline $\begin{array}{l}\text { Sample } \\
\text { number }\end{array}$ & $\begin{array}{l}\text { Sample } \\
\text { name }\end{array}$ & $\begin{array}{l}\text { MLL } \\
\text { state }\end{array}$ & $\begin{array}{l}\text { Leukemia } \\
\text { type }\end{array}$ \\
\hline $\begin{array}{l}1 \\
2 \\
3 \\
4\end{array}$ & $\begin{array}{l}\text { ML-2 } \\
\text { Karpas-45 } \\
\text { RS4;11 } \\
\text { NOMO-1 }\end{array}$ & $\begin{array}{l}\text { AF6 } \\
\text { AFX } \\
\text { AF4 } \\
\text { AF9 }\end{array}$ & $\begin{array}{l}\text { AML } \\
\text { ALL } \\
\text { ALL } \\
\text { AML }\end{array}$ \\
\hline $\begin{array}{l}5 \\
6 \\
7 \\
8\end{array}$ & $\begin{array}{l}\text { HL60 } \\
\text { NT2D1-24h } \\
\text { U937 } \\
\text { MOLT-4 }\end{array}$ & $\begin{array}{l}\text { wt } \\
\text { wt } \\
\text { wt } \\
\text { wt }\end{array}$ & $\begin{array}{l}\text { AML } \\
\text { EC } \\
\text { ALL } \\
\text { ALL }\end{array}$ \\
\hline
\end{tabular}

B

Classification of MLL leukemia type in the test leukemia cell panel

\begin{tabular}{|l|c|c|}
\hline Actual state & $\begin{array}{c}\text { Predicted } \\
\text { fusion }\end{array}$ & $\begin{array}{c}\text { Predicted } \\
\text { wt }\end{array}$ \\
\hline MLL fusion & 4 & 0 \\
\hline MLL wt & 0 & 4 \\
\hline
\end{tabular}

Figure 5 3D-SP correctly classifies MLL leukemia types de novo. (A) Leukemia cell panel used to test 3D-SP. Cell lines are organized by MLL status and leukemia type. (B) Classification results of the test leukemia cell panel by the 3D-SP trained to distinguish between MLL fusion and wt MLL (Figure 1C). Results shown are from de novo classification by the 3D-SP. Matthews Correlation Coefficient (MCC) = 1.0.

below, we examined the changes in transcription among the various leukemias in our study, and found that they do not explain the differences in 5C. Interestingly however, we found that CTCF and cohesin are present at these interacting sites at least in the THP-1 cell line expressing MLL-AF9. CTCF and cohesin are known chromatin organizers [56] and it will be interesting to see whether differences in CTCF or cohesin binding at these sites play a role in the classification.

In the current model of genome structure-function, chromatin conformation and its molecular composition are thought to mutually affect each other to reach and propagate a given activity [33]. One argument, which could arise, therefore is that chromatin conformation accurately classifies leukemia samples because it reflects differences in transcription. However, comparison between classification with chromatin conformation and with gene expression suggests that at least in the case of MLL-fusion derived leukemias, the information provided by the chromatin organization does not simply reflect its transcriptional state. For example, samples that express high levels of 5' end HOXA genes might all classify together because chromatin is likely to be more open in that part of the cluster. We show that this is not the case first by demonstrating that cell samples expressing different levels of HOXA genes classify properly in the same groups, and secondly, by demonstrating that classification is at least comparable or better with chromatin conformation than gene expression. What the classification results suggest is that as a chromatin modifier, the MLL $w t$ and MLL fusion proteins differently affect the molecular composition and conformation of the chromatin in a manner that is at least partly independent of its effect on transcription activity. In turn, our results show that chromatin organization is not merely tissue-specific but can also differ when different diseases affect a given cell type. Long-range transcription regulation might therefore be specifically altered in human diseases, and sometimes even significantly contribute to pathologies.
In the future, it will be interesting to determine whether expanding the $5 \mathrm{C}$ analysis to other MLL fusion target domains can predict leukemia types and subtypes with near-perfect accuracy. Identifying the type and subtype of the leukemia is the most important factor in defining a treatment course, and for this reason it will also be interesting to see whether chromatin conformation can accurately classify samples collected from the blood of patients, and predict overall survival. With the constant improvement of deep sequencing methods and of protocols capturing genome organization $[57,58]$, identifying bona fide chromatin conformation signatures useful in the clinic is becoming increasingly feasible. The clinical importance of biomarkers for diagnosis, therapy selection, and for screening and monitoring disease progression is already appreciated in the treatment of many cancer types [59]. The identification of robust biomarkers will be important in directing patient care towards a customized setting and will require a greater overall understanding of genome regulation including how it is organized in the nuclear space.

\section{Materials and methods}

\section{Ethical approval}

No ethical approval was needed for this study.

\section{Cell culture and description of the $5 \mathrm{C}$ datasets}

All experiments presented in this study were performed using actively growing cells (log-phase) as defined by growth curves. All cells were grown at $37^{\circ} \mathrm{C}$ in $5 \% \mathrm{CO}_{2}$ atmosphere. A complete description of all the cell samples can be found in Additional file 1: Supplementary Materials and methods. The normalized $5 \mathrm{C}$ data in matrix format are found in Additional file 7.

Quantitative real-time polymerase chain reaction (RT-qPCR) HOXA gene expression was measured by quantitative real-time polymerase chain reaction and is shown relative to actin. The RNeasy ${ }^{\odot}$ Mini kit (Qiagen) was used 
to extract total RNA from the samples as described by the manufacturer. The samples were then treated with DNAseI (NEB), and re-purified on Qiagen columns. Two micrograms of the resulting total RNA was used in each reverse transcription reaction with the Superscript ${ }^{\circ}$ III reverse transcriptase kit $\left(\right.$ Invitrogen $\left.^{\mathrm{Tw}}\right)$ and oligo $(\mathrm{dT})_{20}$. The SsoFast ${ }^{\text {tax }}$ EvaGreen $^{(0}$ Supermix (Bio-Rad) was used to quantify the samples by RT-qPCR with a Bio-Rad CFX96 ${ }^{\text {max }}$ $\left(\mathrm{C} 1000^{\mathrm{m}}\right.$ series) real-time system. Quantification was performed by two-fold serial dilutions of total cDNA. The size and specificity of amplified products was verified on agarose gels containing $0.5 \mu \mathrm{g} / \mathrm{mL}$ ethidium bromide and/ or by verifying the melting temperatures of PCR amplicons. An AlphaImager ${ }^{\odot}$ HP coupled to a 12-bit digital camera and equipped with the AlphaView ${ }^{\circ}$ image acquisition and analysis software (version 3.0; Alpha Innotech Corporation) was used to document and analyze the gels. All RT-qPCR primer sequences used in this study were previously described [38], and their sequences are also available on our website [60].

\section{Chromosome conformation capture $(3 \mathrm{C})$ and $3 \mathrm{C}$-carbon copy (5C)}

The chromosome conformation capture $(3 \mathrm{C})$ and $3 \mathrm{C}$ carbon copy (5C) techniques were used as previously described to characterize the chromatin organization of a region containing the HOXA gene cluster [38,51]. The experimental design and the procedure used to generate our 3C and 5C datasets are described in detail in Additional file 1: Supplementary Materials and methods.

\section{Interaction frequency (IF) normalization}

We modified the previously published approach to normalize interaction frequency data [51] to account for the amount of DNA hybridized onto arrays (hybridization efficiency) and improve correction for primer pair efficiencies. This approach is described in detail in Additional file 1: Supplementary Materials and methods, and is illustrated in Additional file 3: Figure S1.

\section{Support vector machine implementation}

A support vector machine (SVM) classifier [53] was implemented using the open-source Weka Java package $[61,62]$. The SVM hyperparameters (notably the soft margin penalty and the RBF width) were fixed for all experiments and the SVM used a polynomial kernel $\left(K(x, y)=\langle x, y\rangle^{p}\right)$ and was trained using sequential optimization minimization, with 1-vs-1 pairwise classification for multi-class problems $[63,64]$. Each $5 \mathrm{C}$ dataset was represented as a single vector of normalized IF values obtained by measuring pairwise chromatin interactions in vivo. The SVM was trained and evaluated using a leave-one-out crossvalidation approach to obtain a classifier with a maximummargin hyperplane in the transformed feature space. The predictive power of individual features was evaluated using the information gain score (also computed using Weka), which measures the reduction of the entropy of the class distribution when the feature is considered. Contacts with large information gains confer the highest amount of predictive power. Informative contacts were selected by determining if the IF values were statistically different between sample types. This was done by calculating $P$ values using a two-sided Student's t-Test in R [65]. The two samples (MLL fusions $v s$. $w t$, or MLL-AF9 $v s$. MLL-ENL) t-test was performed for each feature independently, and features that were retained had reported $P$ values below a threshold of $0.01(P<0.01)$.

The Matthews Correlation Coefficient (MCC) was calculated as a measure of the quality of the classifications performed by the SVM, with values close to +1 indicating perfect prediction, values close to 0 indicating random prediction, and values close to -1 indicating complete disagreement between the prediction and the observation. The MCC statistic was calculated from the confusion matrix as:

$$
\begin{aligned}
& \mathrm{MCC}=\frac{((\mathrm{TP} \times \mathrm{TN})-(\mathrm{FP} \times \mathrm{FN}))}{\mathrm{sqrt}((\mathrm{TP}+\mathrm{FP}) \times(\mathrm{TP}+\mathrm{FN}) \times(\mathrm{TN}+\mathrm{FP}) \times(\mathrm{TN}+\mathrm{FN}))} \\
& \text { where TP : True Positives, TN : True Negatives, FP : False Positives, } \\
& \text { FN : False Negatives }
\end{aligned}
$$

\section{ChIP-seq}

Chromatin immunoprecipitation was conducted as described previously [55] with $5 \mu \mathrm{L}$ of antibodies against CTCF (Millipore, cat. no. 07-729) or Rad21 (Abcam, cat. no. ab992). For Rad21 samples, the BARCODE adaptors were used for generating the BARCODE ChIPseq libraries (BARCODE adaptor [66]). ChIP-seq libraries were sequenced with an Illumina Genome Analyzer (GAiiX) DNA sequencer (36 bps reads, CTCF and WCE) and Illumina Hiseq2000 (51 bps reads, $\operatorname{Rad} 21)$ at the Génome Québec Innovation Centre [67].

\section{ChIP-seq data analysis}

For BARCODE ChIP-seq libraries (Rad21), FASTXToolkit (version 0.0.13) [68] was used to split the reads according to the specific nucleotide barcode in first $4 \mathrm{bp}$ of sequence reads. Before mapping to the genome, the first 4 bps barcode nucleotide of each read were trimmed. Sequence reads with low quality in 3' ends were trimmed to $25 \mathrm{bps}$ (one lane of sequence reads in Rad21 samples). Sequence reads (Fastq format) were aligned to the human genome (UCSC hg19) using the Bowtie program [69]. One mismatch was allowed to the unique mapped reads (option: -v 1 -m 1). Peak calling was performed with the HOMER program [70] and WCE data was used as control. The total mapped tags in each sample were normalized to 'Tags per 10 millions' 
reads. The CTCF or Rad21 binding regions with twofold (CTCF) or four fold (Rad21) over in both control (whole cell extract) and local region (10 kbp) were identified by HOMER program (FDR $<0.001$ ('findpeak' command)). The data was visualized in the hg19 genome version on the UCSC browser.

\section{Databases and URLs}

The data and source code of our software are available through our website with instructions at the following address: [71]. The source code of our software is also attached to this manuscript for archival purpose only (Additional file 8). The 5C and ChIP-seq data for CTCF and $\operatorname{Rad} 21$ can be downloaded from the Gene Expression Omnibus (GEO) website [72].

\section{Additional files}

\section{Additional file 1: Supplementary Materials and methods,}

Supplementary Figure legends, and Supplementary References. The Supplementary Materials and methods contain a detailed description of the cell lines analyzed in this study. It also describes the $3 \mathrm{C}$ and $5 \mathrm{C}$ protocols used to capture chromatin organization, and the computational approach developed to calculate normalized interaction frequencies (IFs). This file also contains the figure legends for (Additional file 3: Figures S1, Additional file 4: Figures S2, Additional file 5: Figures S4, and Additional file 6: Figures $\mathrm{S3}$ respectively), as well as References relevant to Additional file 1.

Additional file 2: Table S1. HOXA gene expression in leukemia cell samples used in this study. This table contains the mRNA quantification of all HOXA genes by RT-qPCR in cell lines used to compare classification by gene expression and chromatin conformation (Additional file 5: Figure S4).

Additional file 3: Figure S1. Workflow of 5C data processing, normalization, and conversion into interaction frequencies. This figure illustrates our approach to calculate normalized IFs from $5 \mathrm{C}$ data. This method and Additional file 3: Figure S1 are described in detail in (Additional file 1: Supplementary Materials and methods and Additional file 3: Figure S1)

Additional file 4: Figure S2. 5 C datasets generated for the 3D-SP training set. This figure shows the $5 \mathrm{C}$ data of the cell samples from the training set in the form of heatmaps as described in Additional file 4: Figure S2 (Additional file 1)

Additional file 5: Figure S4. 3D-SP performs better than gene expression to classify MLL leukemia types. This figure compares classification of leukemia cell samples using HOXA gene expression and HOXA chromatin organization. The figure is described in the Additional file 5: Figure 54 in Additional file 1.

Additional file 6: Figure S3. $5 \mathrm{C}$ datasets generated for the 3D-SP test set. This figure shows the $5 \mathrm{C}$ data of the cell samples from the test set in the form of heatmaps as described in Additional file 6: Figure S3 (Additional file 1).

\section{Additional file 7: This file contains the normalized $5 \mathrm{C}$ data in} matrix format of all the samples produced for this study. These files can be uploaded directly in the my5C platform (http://my5c.umassmed. edu/welcome/welcome.php) [73].

Additional file 8: This file contains the source code for all the software used in this study.

\section{Abbreviations}

3C: Chromosome conformation capture; 5C: 3C-carbon-copy; SVM: Support vector machine.

\section{Competing interests}

The authors declare that they have no competing interests.

\section{Authors' contributions}

MR designed, developed, and carried out the computational analysis, and helped to draft the manuscript. MAF participated in designing the $5 \mathrm{C}$ experiments, produced part of the $5 \mathrm{C}$ datasets, and participated in the mRNA quantification by real-time quantitative PCR. JC carried out part of the $5 C$ experiments, participated in the quantification of mRNAs by real-time quantitative $P C R$, and helped to draft the manuscript. XQDW participated in generating part of the $5 \mathrm{C}$ experiments, and in the quantification of mRNAs by real-time quantitative PCR. HM performed the ChIP-seq experiments and their analysis. MB participated in the design and coordination of the study, and helped to draft the manuscript. JD conceived the study, participated in its design and coordination, and drafted the manuscript. All authors read and approved the final manuscript

\section{Acknowledgements}

We thank Denis Paquette for excellent technical assistance and members of our laboratories for insightful discussions. This work was supported by grants from the Canadian Institutes of Health Research (CIHR MOP-86716), the Terry Fox Foundation (grant \#19252), and an innovation grant from the Canadian Cancer Society (CCS; grant \#701356) to JD, and by a National Sciences and Engineering Research Council (NSERC) Discovery grant to MB. MR was supported by a scholarship from NSERC. MAF was supported by a fellowship from the CCS. XQDW was supported by a scholarship from the Cole Foundation. Funding for open access charge: CCS grant \#701356. JD is a CIHR New Investigator and FRSQ Research Scholar (Fonds de la Recherche en Santé du Québec).

Received: 1 February 2014 Accepted: 30 April 2014

Published: 30 April 2014

\section{References}

1. Dostie J, Bickmore WA: Chromosome organization in the nucleus charting new territory across the Hi-Cs. Curr Opin Genet Dev 2012, 22:125-131.

2. Fraser $P$, Bickmore $W$ : Nuclear organization of the genome and the potential for gene regulation. Nature 2007, 447:413-417.

3. Babu MM, Janga SC, de Santiago I, Pombo A: Eukaryotic gene regulation in three dimensions and its impact on genome evolution. Curr Opin Genet Dev 2008, 18:571-582.

4. Cook PR: A model for all genomes: the role of transcription factories. J Mol Biol 2009, 395:1-10.

5. Pombo A, Branco MR: Functional organisation of the genome during interphase. Curr Opin Genet Dev 2007, 17:451-455.

6. Cremer T, Cremer M: Chromosome territories. Cold Spring Harb Perspect Biol 2010, 2:a003889.

7. Eskiw CH, Cope NF, Clay I, Schoenfelder S, Nagano T, Fraser P: Transcription factories and nuclear organization of the genome. Cold Spring Harb Symp Quant Biol 2010, 75:501-506.

8. Razin SV, Gavrilov AA, Pichugin A, Lipinski M, larovaia OV, Vassetzky YS: Transcription factories in the context of the nuclear and genome organization. Nucleic Acids Res 2011, doi:10.1093/nar/gkr683.

9. $L i G$, Reinberg D: Chromatin higher-order structures and gene regulation. Curr Opin Genet Dev 2011, 21:175-186.

10. West AG, Fraser P: Remote control of gene transcription. Hum Mol Genet 2005, 14:R101-R111.

11. Woodcock CL: Chromatin architecture. Curr Opin Struct Biol 2006, 16:213-220.

12. Gondor A, Ohlsson R: Chromosome crosstalk in three dimensions. Nature 2009, 461:212-217.

13. Tolhuis B, Palstra RJ, Splinter E-g, Grosveld F, de Laat W: Looping and interaction between hypersensitive sites in the active beta-globin locus. Mol Cell 2002, 10:1453-1465.

14. Mishiro T, Ishihara K, Hino S, Tsutsumi S, Aburatani H, Shirahige K, Kinoshita $Y$, Nakao M: Architectural roles of multiple chromatin insulators at the human apolipoprotein gene cluster. EMBO J 2009, 28:1234-1245.

15. Morey C, Avner P: The demoiselle of X-inactivation: 50 years old and as trendy and mesmerising as ever. PLoS Genet 2011, 7:e1002212.

16. Chaumeil J, Le Baccon P, Wutz A, Heard E: A novel role for Xist RNA in the formation of a repressive nuclear compartment into which genes are recruited when silenced. Genes Dev 2006, 20:2223-2237.

17. Mattout A, Pike BL, Towbin BD, Bank EM, Gonzalez-Sandoval A, Stadler MB, Meister P, Gruenbaum Y, Gasser SM: An EDMD mutation in C. elegans 
lamin blocks muscle-specific gene relocation and compromises muscle integrity. Curr Biol 2011, 21:1603-1614.

18. Pomerantz MM, Ahmadiyeh N, Jia L, Herman P, Verzi MP, Doddapaneni $H_{\text {, }}$ Beckwith CA, Chan JA, Hills A, Davis M, Yao K, Kehoe SM, Lenz HJ, Haiman CA, Yan C, Henderson BE, Frenkel B, Barretina J, Bass A, Tabernero J, Baselga J, Regan MM, Manak JR, Shivdasani R, Coetzee GA, Freedman ML: The 8q24 cancer risk variant rs6983267 shows long-range interaction with MYC in colorectal cancer. Nat Genet 2009, 41:882-884.

19. Pittman AM, Naranjo S, Jalava SE, Twiss P, Ma Y, Olver B, Lloyd A, Vijayakrishnan J, Qureshi M, Broderick P, van Wezel T, Morreau H, Tuupanen S, Aaltonen LA, Alonso ME, Manzanares M, Gavilan A, Visakorpi T, Gomez-Skarmeta JL, Houlston RS: Allelic variation at the $8 \mathrm{q} 23.3$ colorectal cancer risk locus functions as a cis-acting regulator of EIF3H. PLoS Genet 2010, 6:e1001126.

20. Meyer KB, Maia AT, O'Reilly M, Ghoussaini M, Prathalingam R, Porter-Gill P, Ambs S, Prokunina-Olsson L, Carroll J, Ponder BA: A functional variant at a prostate cancer predisposition locus at $8 \mathrm{q} 24$ is associated with PVT1 expression. PLoS Genet 2011, 7:e1002165.

21. Tiwari VK, Cope L, McGarvey KM, Ohm JE, Baylin SB: A novel 6 C assay uncovers polycomb-mediated higher order chromatin conformations. Genome Res 2008, 18:1171-1179.

22. Erfurth F, Hemenway CS, de Erkenez AC, Domer PH: MLL fusion partners AF4 and AF9 interact at subnuclear foci. Leukemia 2004, 18:92-102.

23. Tolhuis B, Blom M, Kerkhoven RM, Pagie L, Teunissen H, Nieuwland M, Simonis M, de Laat W, van Lohuizen M, van Steensel B: Interactions among polycomb domains Are guided by chromosome architecture. PLOS Genet 2011, 7:e1001343.

24. Smith E, Lin C, Shilatifard A: The super elongation complex (SEC) and MLL in development and disease. Genes Dev 2011, 25:661-672.

25. Balgobind BV, Zwaan CM, Pieters R, Van den Heuvel-Eibrink MM: The heterogeneity of pediatric MLL-rearranged acute myeloid leukemia. Leukemia 2011, 25:1239-1248.

26. Tkachuk DC, Kohler S, Cleary ML: Involvement of a homolog of drosophila trithorax by 11q23 chromosomal translocations in acute leukemias. Cell 1992, 71:691-700

27. Thirman MJ, Gill HJ, Burnett RC, Mbangkollo D, McCabe NR, Kobayashi H, Ziemin-van Der Peol S, Kaneko Y, Morgan R, Sandberg AA, Chaganti RSK, Larson RA, Le Beau MM, Diaz MO, Rowley JD: Rearrangement of the MLL gene in acute lymphoblastic and acute myeloid leukemias with 11 q23 chromosomal translocations. N Engl J Med 1993, 329:909-914.

28. Daser A, Rabbitts TH: Extending the repertoire of the mixed-lineage leukemia gene MLL in leukemogenesis. Genes Dev 2004, 18:965-974.

29. Ferrando AA, Armstrong SA, Neuberg DS, Sallan SE, Silverman LB, Korsmeyer SJ, Look AT: Gene expression signatures in MLL-rearranged T-lineage and B-precursor acute leukemias: dominance of HOX dysregulation. Blood 2003, 102:262-268.

30. Lin C, Smith ER, Takahashi H, Lai KC, Martin-Brown S, Florens L, Washburn MP, Conaway JW, Conaway RC, Shilatifard A: AFF4, a component of the ELL/P-TEFb elongation complex and a shared subunit of MLL chimeras, can link transcription elongation to leukemia. Mol Cell 2010, 37:429-437.

31. Biswas D, Milne TA, Basrur V, Kim J, Elenitoba-Johnson KS, Allis CD, Roeder RG: Function of leukemogenic mixed lineage leukemia 1 (MLL) fusion proteins through distinct partner protein complexes. Proc Natl Acad Sci U S A 2011, 108:15751-15756.

32. Marschalek R: Mechanisms of leukemogenesis by MLL fusion proteins. Br J Haematol 2011, 152:141-154.

33. Cavalli G, Misteli T: Functional implications of genome topology. Nat Struct Mol Biol 2013, 20:290-299.

34. Look AT: Oncogenic transcription factors in the human acute leukemias. Science 1997, 278:1059-1064.

35. Liu H, Cheng EH, Hsieh JJ: MLL fusions: pathways to leukemia. Cancer Biol Ther 2009, 8:1204-1211.

36. Cowell IG, Sondka Z, Smith K, Lee KC, Manville CM, Sidorczuk-Lesthuruge M, Rance HA, Padget K, Jackson GH, Adachi N, Austin CA: Model for MLL translocations in therapy-related leukemia involving topoisomerase Ilbeta-mediated DNA strand breaks and gene proximity. Proc Natl Acad Sci U S A 2012, 109:8989-8994

37. Eguchi M, Eguchi-Ishimae M, Greaves M: Molecular pathogenesis of MLL-associated leukemias. Int J Hematol 2005, 82:9-20.

38. Fraser J, Rousseau M, Shenker S, Ferraiuolo MA, Hayashizaki Y, Blanchette M, Dostie J: Chromatin conformation signatures of cellular differentiation. Genome Biol 2009, 10:R37.
39. Sauvageau G, Lansdorp PM, Eaves CJ, Hogge DE, Dragowska WH, Reid DS, Largman C, Lawrence HJ, Humphries RK: Differential expression of homeobox genes in functionally distinct CD34+ subpopulations of human bone marrow cells. Proc Natl Acad Sci U S A 1994, 91:12223-12227.

40. Pineault N, Helgason CD, Lawrence HJ, Humphries RK: Differential expression of Hox, Meis1, and Pbx1 genes in primitive cells throughout murine hematopoietic ontogeny. Exp Hematol 2002, 30:49-57.

41. Calvo R, West J, Franklin W, Erickson P, Bemis L, Li E, Helfrich B, Bunn P, Roche J, Brambilla E, Rosell R, Gemmill RM, Drabkin HA: Altered HOX and WNT7A expression in human lung cancer. Proc Natl Acad Sci U S A 2000, 97:12776-12781.

42. Makiyama K, Hamada J, Takada M, Murakawa K, Takahashi Y, Tada M, Tamoto E, Shindo G, Matsunaga A, Teramoto K, Komuro K, Kondo S, Katoh $\mathrm{H}$, Koike T, Moriuchi T: Aberrant expression of HOX genes in human invasive breast carcinoma. Oncol Rep 2005, 13:673-679.

43. Maeda K, Hamada J, Takahashi Y, Tada M, Yamamoto Y, Sugihara T, Moriuchi T: Altered expressions of HOX genes in human cutaneous malignant melanoma. Int J Cancer 2005, 114:436-441.

44. Eklund EA: The role of HOX genes in malignant myeloid disease. Curr Opin Hematol 2007, 14:85-89.

45. Thorsteinsdottir U, Sauvageau G, Hough MR, Dragowska W, Lansdorp PM, Lawrence HJ, Largman C, Humphries RK: Overexpression of HOXA10 in murine hematopoietic cells perturbs both myeloid and lymphoid differentiation and leads to acute myeloid leukemia. Mol Cell Biol 1997, 17:495-505.

46. Kroon E, Krosl J, Thorsteinsdottir U, Baban S, Buchberg AM, Sauvageau G Hoxa9 transforms primary bone marrow cells through specific collaboration with Meis1a but not Pbx1b. Embo J 1998, 17:3714-3725.

47. Ayton PM, Cleary ML: Transformation of myeloid progenitors by MLL oncoproteins is dependent on Hoxa7 and Hoxa9. Genes Dev 2003, 17:2298-2307

48. Garcia-Fernandez J: The genesis and evolution of homeobox gene clusters. Nat Rev Genet 2005, 6:881-892.

49. Amores A, Force A, Yan YL, Joly L, Amemiya C, Fritz A, Ho RK, Langeland J, Prince V, Wang YL, Westerfield M, Ekker M, Postlethwait JH: Zebrafish hox clusters and vertebrate genome evolution. Science 1998, 282:1711-1714.

50. Ethier SD, Miura $\mathrm{H}$, Dostie J: Discovering genome regulation with $3 \mathrm{C}$ and 3C-related technologies. Biochim Biophys Acta 1819, 2012:401-410.

51. Dostie J, Richmond TA, Arnaout RA, Selzer RR, Lee WL, Honan TA, Rubio ED, Krumm A, Lamb J, Nusbaum C, Green RD, Dekker J: Chromosome Conformation Capture Carbon Copy (5C): a massively parallel solution for mapping interactions between genomic elements. Genome Res 2006, 16:1299-1309.

52. Ferraiuolo MA, Rousseau M, Miyamoto C, Shenker S, Wang XQ, Nadler M, Blanchette M, Dostie J: The three-dimensional architecture of Hox cluster silencing. Nucleic Acids Res 2010, 38:7472-7484.

53. Cortes C, Vapnik V: Support-vector networks. Mach Learn 1995, 20:273-297.

54. Noble WS: What is a support vector machine? Nat Biotechnol 2006, 24:1565-1567.

55. Rousseau M, Crutchley JL, Miura H, Suderman M, Blanchette M, Dostie J: Hox in motion: tracking HoxA cluster conformation during differentiation. Nucleic Acids Res 2014, 42:1524-1540.

56. Phillips JE, Corces VG: CTCF: master weaver of the genome. Cell 2009, 137:1194-1211.

57. Fraser J, Ethier SD, Miura H, Dostie J: A Torrent of data: mapping chromatin organization using $5 \mathrm{C}$ and high-throughput sequencing. Methods Enzymol 2012, 513:113-141.

58. Ferraiuolo MA, Sanyal A, Naumova N, Dekker J, Dostie J: From cells to chromatin: capturing snapshots of genome organization with $5 \mathrm{C}$ technology. Methods 2012, 58:255-267.

59. Ludwig JA, Weinstein JN: Biomarkers in cancer staging, prognosis and treatment selection. Nat Rev Cancer 2005, 5:845-856.

60. Dr Josee Dostie Laboratory. [http://Dostielab.biochem.mcgill.ca]

61. Weka 3: Data Mining Software in Java, version 3.7.5. [www.cs.waikato.ac. $\mathrm{nz} / \mathrm{ml} /$ weka/]

62. Hall M, Frank E, Holmes G, Pfahringer B, Reutemann P, Witten $\mid H$ : The WEKA data mining software: an update. SIGKDD Explorations 2009, 11:10-18.

63. Keerthi SS, Shevade SK, Bhattacharyya C, Murthy KRK: Improvements to Platt's SMO algorithm for SVM classifier design. Neural Computation 2001, 13:13.

64. Hastie T, Tibshirani R: Classification by pairwise coupling. Ann Statist 1998, 26:451-800 
65. R: a language and environment for statistical computing. $R$ foundation for statistical computing, Vienna, Austria. [http://www.R-project.org/]

66. Lefrancois P, Euskirchen GM, Auerbach RK, Rozowsky J, Gibson T, Yellman CM, Gerstein M, Snyder M: Efficient yeast ChIP-Seq using multiplex short-read DNA sequencing. BMC Genomics 2009, 10:37.

67. The McGill University and Génome Québec Innovation Centre. [http:// gqinnovationcenter.com/]

68. Collection of command line tools for Short-Reads FASTA/FASTQ files preprocessing. http://hannonlab.cshl.edu/fastx_toolkit/.

69. Langmead B, Trapnell C, Pop M, Salzberg SL: Ultrafast and memory-efficient alignment of short DNA sequences to the human genome. Genome Biol 2009, 10:R25.

70. Software for motif discovery and ChIP-Seq analysis. http://homer.salk.edu/ homer/chipseq/.

71. Dr. Josee Dostie Laboratory. http://dostielab.biochem.mcgill.ca/research.htm.

72. $5 \mathrm{C}$ and ChIP-seq datasets generated for this study downloadable from the Gene Expression Omnibus. http://www.ncbi.nlm.nih.gov/geo/query/acc.cgi? acc $=$ GSE55408.

73. Lajoie BR, van Berkum NL, Sanyal A, Dekker J: My5C: web tools for chromosome conformation capture studies. Nat Methods 2009, 6:690-691.

doi:10.1186/gb-2014-15-4-r60

Cite this article as: Rousseau et al:: Classifying leukemia types with

chromatin conformation data. Genome Biology 2014 15:R60.

\section{Submit your next manuscript to BioMed Central and take full advantage of:}

- Convenient online submission

- Thorough peer review

- No space constraints or color figure charges

- Immediate publication on acceptance

- Inclusion in PubMed, CAS, Scopus and Google Scholar

- Research which is freely available for redistribution 